\title{
Closed Traumatic Rupture of Tibialis Anterior Tendon
}

\author{
Hui Chen*, Gang-Rui Jia and Xin-Qiang Wang \\ Department of Orthopaedics, BenQ Medical Center, The Affiliated BenQ Hospital of Nanjing Medical University, Nanjing, Jiangsu \\ Province, China
}

*Corresponding author: Hui Chen, Department of Orthopaedics, BenQ Medical Center, The Affiliated BenQ Hospital of Nanjing

Medical University, Nanjing, Jiangsu Province, China

\begin{tabular}{|c|c|}
\hline ARTICLE INFO & ABSTRACT \\
\hline Received: 慧 March 10, 2021 & $\begin{array}{l}\text { Citation: Hui Chen, Gang-Rui Jia, Xin-Qiang Wang. Closed Traumatic Rupture of Tibialis } \\
\text { Anterior Tendon. Biomed J Sci \& Tech Res 34(5)-2021. BJSTR. MS.ID.005607. }\end{array}$ \\
\hline
\end{tabular}

\section{Introduction}

Closed rupture of Tibialis Anterior Tendon (TAT) is very uncommon. It not only be related to trauma, but also occurs spontaneously in tendons weakened by underlying chronic diseases, such as psoriatic arthritis, diabetes, gout, and local corticoid injection [1]. For a variety of reasons, early diagnosis of the closed traumatic rupture of the TAT is challenging We hear report such a closed TAT rupture combined with multiple fractures of ribs and discuss diagnosis and treatment of such a condition.

\section{Case Report}

A 50-year-old male patient came to the clinics with a chief complaint of weakened dorsal extension of the right foot and steppage gait. More than a month ago, he stumbled over the staires and felt an intense pain on his chest [2]. As the chest pain faded, he gradually experienced extension weakness in his right ankle, with occasional walking pain. Physical examination revealed mild swelling of the anteromedial malleolus. A mobile and painful soft mass could be touched at the joint level, while the most distal part of TAT was vacant [3]. Muscle strength of the ankle extensors remained grade 4 while the angle of ankle extension was partially lost (15 degrees). The extensor hallucis longus played the role of extensor substitution of the right foot. Silfverskiöld test revealed no contracture of the gastrocnemius. American Orthopaedic Foot \& Ankle Society (AOFAS) Scores of the hind foot and ankle was 52. Approximately 6 weeks later, ultrasound of the right ankle was carried out after the trauma, and the report was subcutaneous hematoma (Figure 1). At our clinic, $5 \mathrm{ml}$ light ischemic liquid was obtained from the pain point of right anterior ankle by manual syringe, then local area was compressed, but the "hematoma" returned soon. MRI performed showed a complete tear of the TAT, with the TAT proximally retracted and agglomerated, with some residual edema in the anterior ankle (Figure 2).

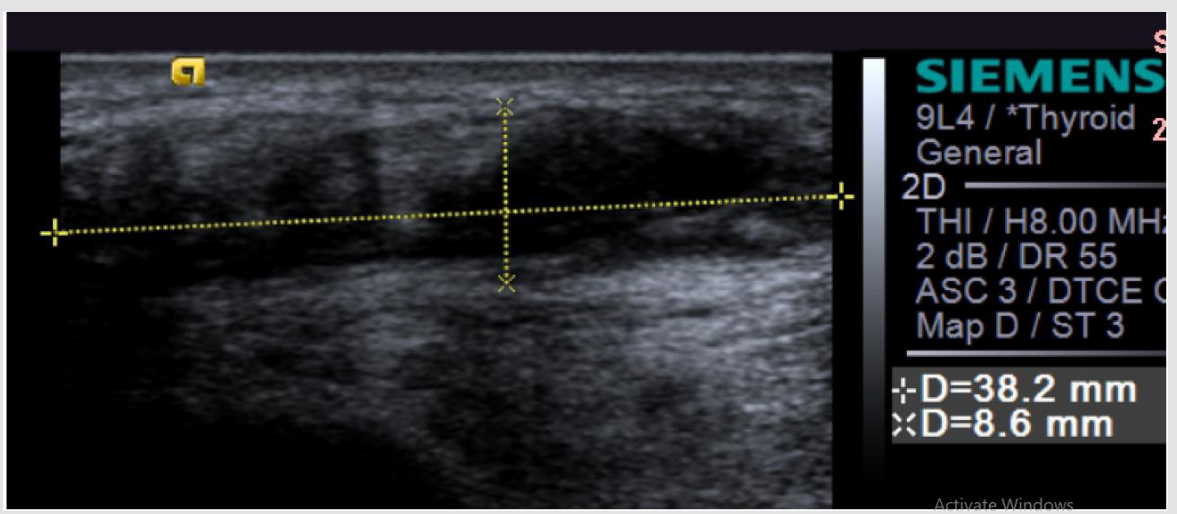

Figure 1: Ultrasound of the painful area of the right ankle showed a $38 \mathrm{~mm} \times 9 \mathrm{~mm}$ cystic and solid mixing signals (subcutaneous hematoma). 


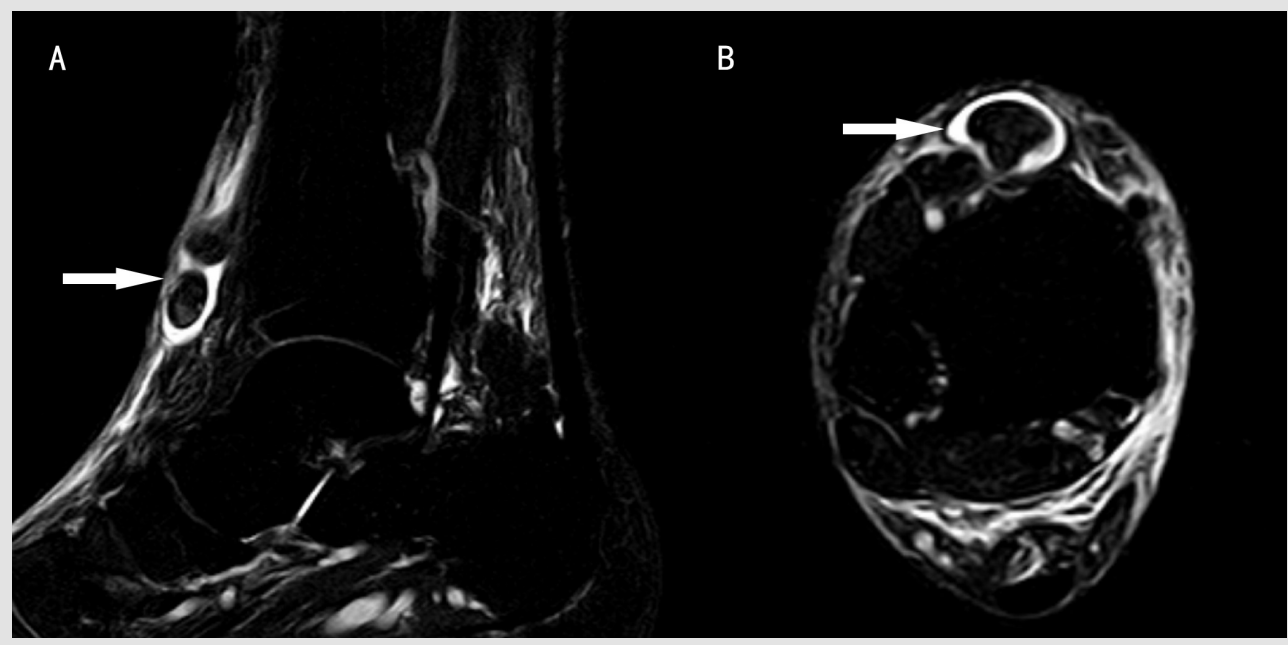

Figure 2: MRI of the right ankle

a) Sagital view of the T2-weighted images showed the retracted, agglomerated proximal end of the TAT (arrow).

b) Axial view showed low signals of the thickened, retracted proximal portion of TAT (arrow).

Delayed repair of the ruptured tendon was performed 42 days after the injury. An anteromedial approach along the route of the tendon was used. Exploration revealed that the inferior extensor retinaculum was partially broken, and the TAT ruptured from its insertion of cuneiform with the proximal stump retracted. There was no obvious pathological change in the end of the ruptured tendon. After an careful debridement, a mild defect about $1.0 \mathrm{~cm}$ appeared at the insertion point of the tendon [4-7]. Silfverskiöld test was again performed to evaluate the contracture of gastrocnemius (Figure 3). After the original insertion point was decortexed the TAT was fixed anatomically $5 \mathrm{~mm}$ suture anchor (Figure 4). Meanwhile, medial surface of the scaphoid was decortexed, and the tendon was fixed consecutively on the scaphoid by a $1.0 \mathrm{~cm}$ spiked staple (Figure 5). Inferior extensor retinaculum was repaired and the wound was then closed in layers with absorbable sutures. The ankle was immobilized in a short leg cast in maximum ankle extension for 6 weeks followed by protecting brace for another month. After the removal of the cast, rehabilitation was practiced [8]. Two and half months after surgery, the patient returned to full weight bearing. Three months after surgery, his activity level of the right ankle was slightly less than previously, and there was a mild slapping gait. He denied pain in his right foot. Physical examination revealed that the tendon was palpable along its normal course and there was no tenderness. The AOFAS Scores was improved to 77.

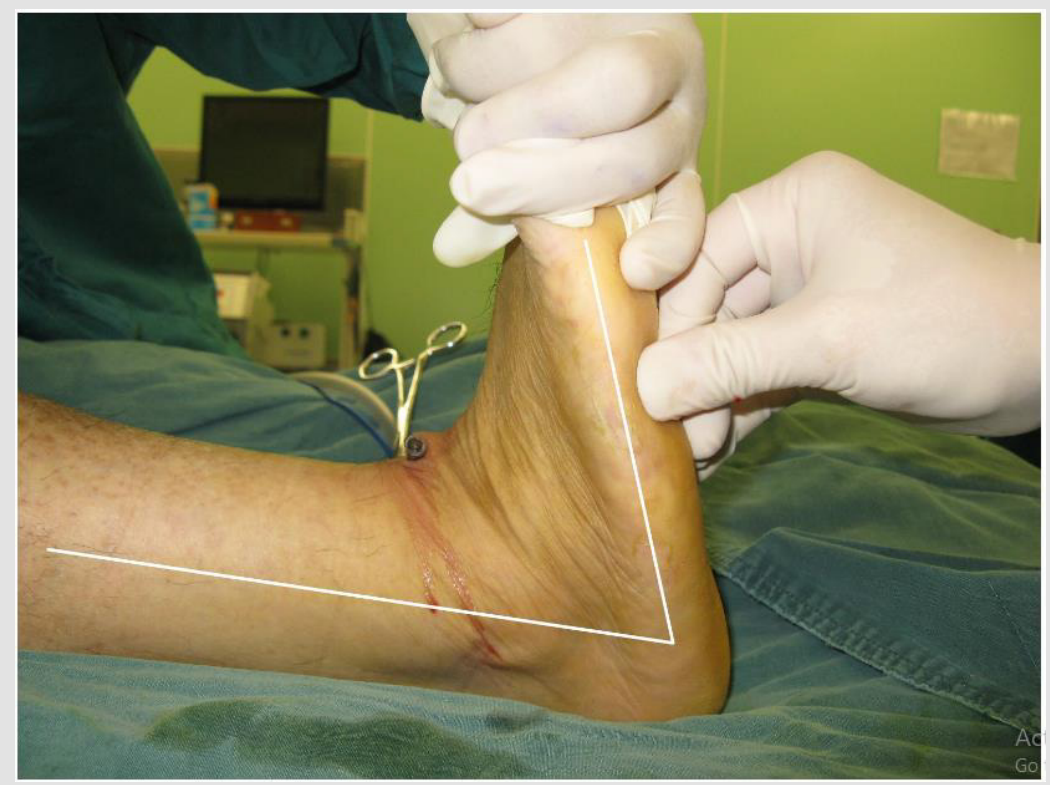

Figure 3: Silfverskiöld test intraoperatively showed that the maximum extension of the ankle was 20 degrees. 


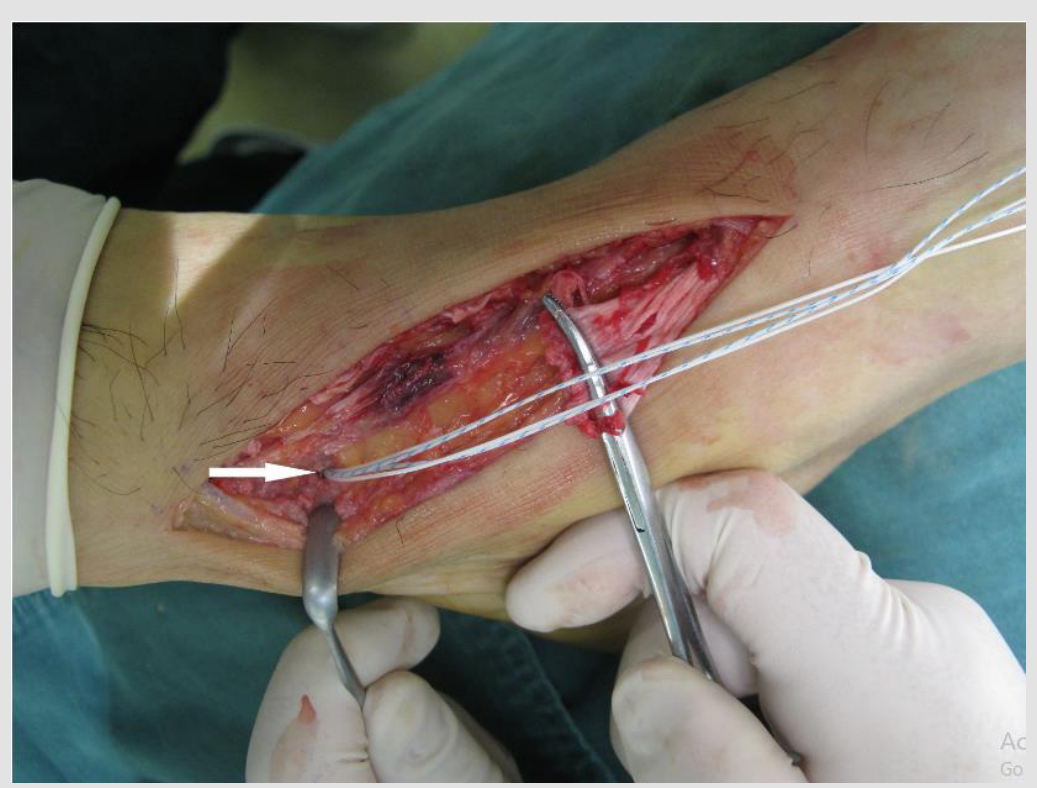

Figure 4: After decortex of the medial cuboid the TAT was fixed on the original insertion point by suture anchor (arrow).

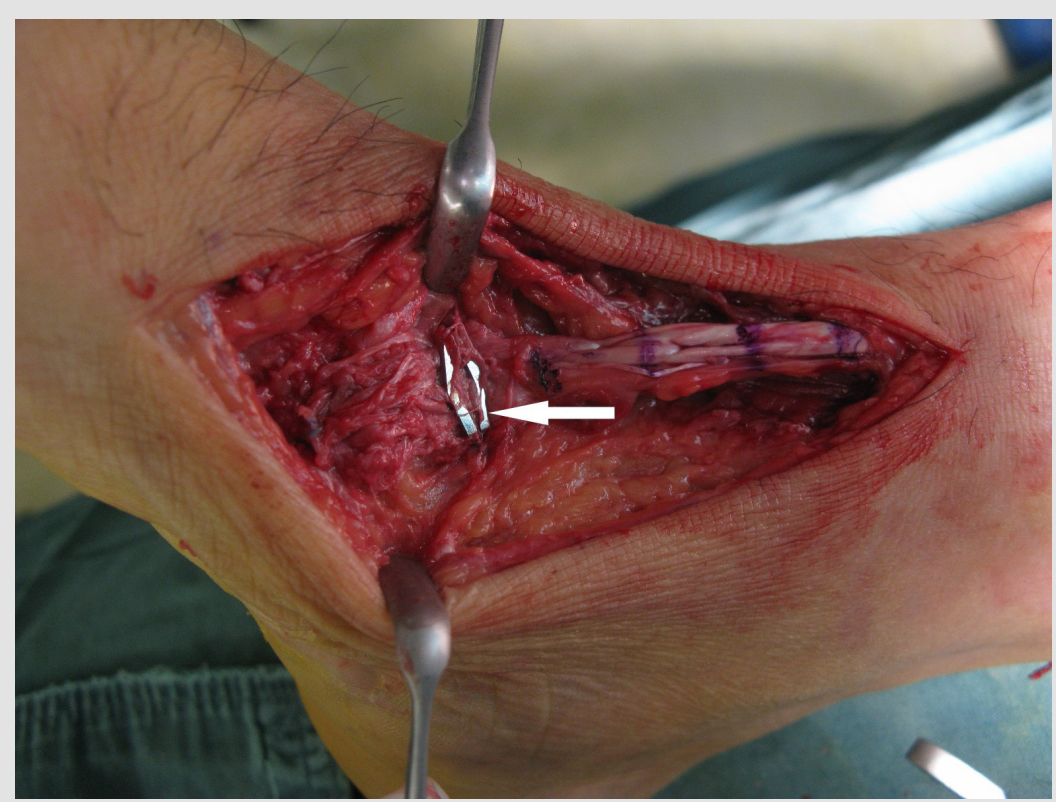

Figure 5: As an accessory fixation, the TAT was attached to the scaphoid anteromedially by a spiked staple (arrow).

\section{Discussion}

The tibialis anterior muscle is the major extensors of the ankle. Covered with euangiotic sheathes, the tendon slides underneath the superior and inferior extensor retinaculum and inserts mainly into the medial aspect of the first cuneiform. Blood supply of the tendon tissue comes from the well vascularized peritendinous, and the avascular zone around the superior and inferior retinacula is prone to be ruptured [9]. Due to the existing avascularity, an osseous rupture of the tendon can also occur, but would be rarer in closed rupture. Because of the above mentioned blood supply anatomy, more attention to the intraoperative protection and repair of tendon tissue around is essential. Diagnosis of closed traumatic rupture of the TAT may be difficult. The patients often present a relatively mild pain for weeks to years following the injury [10]. When it is associated with other impairments, the rupture of the TAT is inclined to be neglected, especially with immobilization by cast or brace. Because most of the presentations are nonspecific, such as local swelling, pressing pain and dysfunction etc, early diagnosis of the closed traumatic rupture of the TAT is challenging. Although the extensor hallucis longus can make partial compensation after rupture of the TAT, a few weeks later, some discomforts may become 
obvious, such as extension weakness, foot drop gait slapping of the foot etc. Theoretically, the typical presentations are patients between 50 and 60 years 2 with a complaint of slapping foot or tripping gait, a tender bulbous mass consisting of retractile tendon commonly at the level of the ankle joint, and the palpable vacant of the route of the TAT with maximum ankle extension. As the swelling gradually disappears, the clinical signs may become typically. In spite of this, clinical signs can be easily missed.

There are several imaging examinations of the TAT, including ultrasound, X-ray and MRI. In ultrasound, a mass is usually observed with effusions around TAT. Unfortunately, an inexperienced ultrasound doctor may fail to detect the lesion and give the wrong diagnosis like subcutaneous hematoma. X-rays are mainly used to exclude fractures and make a comparison before and after the operation when some implants are used. MRI is a useful method to confirm and characterize the rupture of TAT [4]. T2-weighted images show edema, disruption and retraction of the TAT at the level of the ankle joint. But MRI is too expensive to be performed early in any potential patients, it is usually done as the last means or preoperative evaluation of a ruptured tendon. Treatments of closed traumatic rupture of the TAT are various. Conservative treatment of TAT rupture has been associated with some late sequelae, including mild to moderate flatfoot, decreased ankle motion, and heel cord contracture [7]. Better immediate and longtime results after surgical repair of the ruptured TAT are reported in several papers. Surgical intervention has shown fewer complications [3].

Although several surgical techniques of for TAT repaire have been reported, anatomic reconstruction is always emphasized Although repair by tendon grafting or free tendon transfer procedures obtains satisfied results, these methods remain additional risk of functional deficiency in the donor area. If the tendon contracture is not evident, primary repair may be accomplished by end-to-end suturing through a suture anchor or fixation through an osseous tunnel. Additional fixation on navicular bone by spiked staple is a way of adding a healing spot and partially decreasing bow-string potency. Decortexing is necessary for suture fixing through an anchor to promote tendon-bone healing. Although possibility of gastrocnemius contracture within 6 weeks is minimal, Silfverskiöld test makes sense in surgical planning. Once contracture of gastrocnemius is confirmed, achilles tendon loosening or lengthening should be performed. Repairs of the peritendineum and extensor retinaculum are very important. Vascularized peritendineum is the main blood supply of TAT, which is also a sliding tissue to prevent adhesion. If the retinaculum is not repaired, bowstringing may occurre, and the strength will be weakened. Some authors [11] claimed that anatomical closure of the extensor retinaculum is associated with adhesion formation and restrictions, the open superior extensor retinaculum may free up the adhesions.

\section{Conclusion}

We report a 50-year-old male patient with the closed traumatic rupture of TAT. Early diagnosis of this condition is very difficult. Early recognization of this rupture is very important for good function. This patient underwent surgical repair 6 weeks after the injury. A good function was obtained, despite the delay in surgical intervention. The authors propose that surgical technique should be performed through anatomy reconstruction as soon as possible.

\section{References}

1. Coughlin MJ (1999) Disorders of tendons surgery of the foot and ankle. In Coughlin MJ, Mann RA (Eds.), ( $7^{\text {th }}$ edn.), St. Louis, Missouri p. 786-99.

2. Dooley BJ, Kudelka P, Menelaus MB (1980) Subcutaneous rupture of the tendon of tibialis anterior. J Bone Joint Surg Br. Nov 62(4): 471-472.

3. Ellington JK, McCormick J, Marion C (2010) Surgical outcome following tibialis anterior tendon repair. Foot Ankle Int. May 31(5): 412-417.

4. Gallo RA, Kolman BH, Daffner RH, Sciulli RL, Roberts CC (2004) MRI of tibialis anterior tendon rupture. Skeletal Radiol. Feb 33(2): 102-106.

5. Griffiths JC (1965) Tendon injuries around the ankle. J Bone Joint Surg Br 47(4): 686-689.

6. Mechrefe AP, Walsh EF, DiGiovanni CW (2006) Anterior tibial tendon avulsion with distal tibial fracture entrapment: case report. Foot Ankle Int 27(8): 645-647.

7. Meyn MA (1975) Closed rupture of the anterior tibial tendon. A case report and review of the literature. Clin Orthop Relat Res 113: 154-157.

8. Patten A, Pun WK (2000) Spontaneous rupture of the tibialis anterior tendon: a case report and literature review. Foot Ankle Int 21(8): 697700 .

9. Petersen W, Stein V, Tillmann B (1999) Blood supply of the tibialis anterior tendon. Arch Orthop Trauma Surg 119(7-8): 371-375.

10. Sammarco VJ, Sammarco GJ, Henning C, Chaim S (2009) Surgical repair of acute and chronic tibialis anterior tendon ruptures. J Bone Joint Surg Am 91(2): 325-332.

11. Wong MW (2004) Traumatic tibialis anterior tendon rupture-delayed repair with free sliding tibialis anterior tendon graft. Injury 35(9): 940944. 
ISSN: 2574-1241

DOI: $10.26717 /$ BJSTR.2021.34.005607

Hui Chen. Biomed J Sci \& Tech Res

(c) (P) This work is licensed under Creative

Submission Link: https://biomedres.us/submit-manuscript.php

$\begin{array}{ll}\text { BIOMEDICAL } & \text { Assets of Publishing with us } \\ \text { RESEARCHES } & \text { - Global archiving of articles } \\ \text { - Immediate, unrestricted online access } & \text { - Rigorous Peer Review Process } \\ & \text { - Authors Retain Copyrights } \\ \end{array}$

